\title{
SÍNTESIS, CARACTERIZACIÓN Y EVALUACIÓN DE LA ACTIVIDAD BIOLÓGICA DE COMPUESTOS DE COORDINACIÓN DE COBALTO CON PIRAZINAMIDA
}

\author{
Natalia Karolina Chávez Llallire ${ }^{\mathrm{a}}$, Rocío Isabel Ramírez Pantia ${ }^{\mathrm{a}}$ Patricia Sheen Cortovarría ${ }^{\mathrm{b}}$, \\ Mirko Juan Zimic Peralta ${ }^{\mathrm{b}}$, Ana Cecilia Valderrama Negrón ${ }^{\mathrm{a}^{*}}$
}

\begin{abstract}
RESUMEN
En este estudio se han sintetizado dos nuevos compuestos de coordinación de cobalto con pirazinamida: Co-PZA (1) y Co-PZA (2), a partir de dos sales precursoras de cobalto y el fármaco pirazinamida. El objetivo de este estudio ha sido elucidar la estructura química de cada complejo sintetizado mediante el uso de las técnicas de caracterización conocidas, así como evaluar y comparar su actividad biológica respecto al fármaco libre pirazinamida. Con respecto a la caracterización, se usaron las técnicas de análisis elemental de $\mathrm{C}, \mathrm{H}$ y N, espectroscopía de absorción atómica de cobalto, análisis termogravimétrico, susceptibilidad magnética, espectroscopía de UV-Visible, infrarrojo y Raman. La actividad biológica fue evaluada determinando la concentración mínima inhibitoria en cultivos de Mycobacterium tuberculosis. La fórmulas moleculares propuestas a partir de los resultados de la caracterización fueron: $\left[\mathrm{Co}(\mathrm{PZA})_{2} \mathrm{Cl}_{2}\right]$ para el complejo Co-PZA (1) y $\left[\mathrm{Co}(\mathrm{PZA})_{2}\left(\mathrm{NO}_{3}\right)_{2}\right] \cdot \mathrm{H}_{2} \mathrm{O}$ para el complejo Co-PZA (2). Los dos complejos presentaron mayor capacidad antimicrobiana que pirazinamida.
\end{abstract}

Palabras clave: cobalto, pirazinamida, tuberculosis.

\section{SYNTHESIS, CARACTERIZATION AND EVALUATION OF BIOLOGICAL ACTIVITY OF COBALT WITH PYRAZINAMIDE COORDINATION COMPOUNDS}

\begin{abstract}
In this study, two new cobalt with pyrazinamide (PZA) coordination compounds: Co-PZA (1) and Co-PZA (2) were synthesized from two cobalt precursor salts. The aim of this study is to elucidate the chemical structure of each synthesized complex by using characterization

\footnotetext{
a Laboratorio de Investigación en Biopolímeros y Metalofármacos (LIBIPMET), Facultad de Ciencias, Universidad Nacional de Ingeniería, Av. Túpac Amaru 210, Rímac, Lima, Perú, anitacvn29@yahoo.com.mx.

b Unidad de Bioinformática y Biología Molecular, Laboratorios de Investigación y Desarrollo, Facultad de Ciencias y Filosofía, Universidad Peruana Cayetano Heredia, Av. Honorio Delgado 430, SMP, Lima, Perú.
} 
techniques, as well as to evaluate their anti-tuberculous activity compared to pyrazinamide. The characterization techniques comprised, $\mathrm{C}, \mathrm{H}$ and $\mathrm{N}$ elemental analysis, atomic absorption spectroscopy of cobalt, thermogravimetric analysis, magnetic susceptibility, UV-Visible, infrared and Raman spectroscopy. The antibiotic activity was tested by determining the minimum inhibitory concentration in a Mycobacterium tuberculosis culture. The predicted molecular formulas from characterization results were: $\left[\mathrm{Co}(\mathrm{PZA})_{2} \mathrm{Cl}_{2}\right]$ for $\mathrm{Co}-\mathrm{PZA}$ (1) complex and $\left[\mathrm{Co}(\mathrm{PZA})_{2}\left(\mathrm{NO}_{3}\right)_{2}\right] \cdot \mathrm{H}_{2} \mathrm{O}$ for Co-PZA (2) complex. The two complexes showed a higher antimicrobial capacity than pyrazinamide.

Key words: cobalt, pyrazinamide, tuberculosis.

\section{INTRODUCCIÓN}

La tuberculosis es una enfermedad causada por Mycobacterium tuberculosis, altamente contagiosa y trasmitida de persona a persona por vía aérea a través de gotas expelidas desde la garganta y pulmones de personas con tuberculosis activas ${ }^{1}$. Un creciente número de personas contraen tuberculosis, afectando cada año a 10 millones de personas y matando a 1,5 millones de ellas. El curso de la enfermedad se ve comprometido, en pacientes con sistemas inmunes comprometidos, ya sea por fármacos inmunosupresores, sustancias en abuso, o SIDA/VIH, afectando especialmente a poblaciones en África y en países asiáticos como China, India o Indonesia ${ }^{2}$. Por lo tanto, la tuberculosis es una de las principales causas actuales de muerte en el mundo.

Otro problema crítico asociado a la tuberculosis ha sido la resistencia a los fármacos, limitando la capacidad del control de la enfermedad, generando la aparición de tuberculosis multidrogo-resistente (TB-MDR) y tuberculosis extremadamente drogo-resistente (TB$\mathrm{XDR})^{3}$.

La pirazinamida (PZA) actúa como bactericida matando los bacilos tuberculosos semilatentes a un $\mathrm{pH}$ ácido que no son destruidos por otras drogas antituberculosas. Se usa en esquemas de tratamiento de primera y segunda línea, y en paticular en combinación con isoniazida, etambutol y rifampicina en diferentes formas de dosificación ${ }^{4}$. La aparición de cepas resistentes a PZA representa un problema importante en la salud pública a nivel mundial. Por tal razón, se tiene la necesidad de identificar nuevos agentes terapéuticos que permitan superar las limitaciones actuales de los esquemas de tratamiento frente a los casos de tuberculosis drogo-resistente.

Se han reportado numerosas investigaciones de complejos metálicos con diferentes fármacos utilizando como ion metálico a $\mathrm{Cu}(\mathrm{II}), \mathrm{Zn}(\mathrm{II}), \mathrm{Fe}(\mathrm{II}), \mathrm{Co}(\mathrm{II}), \mathrm{Ni}(\mathrm{II}), \mathrm{Cd}(\mathrm{II})$, resultando un aumento de la actividad biológica/antimicobiana y disminución en el desarrollo de la resistencia antibiótica ${ }^{4,5}$. Por tal motivo los compuestos antituberculosos obtenidos a partir de la coordinación de un ligando con iones metálicos son potencialmente una mejora en la estrategia para el tratamiento de esta enfermedad. 
Este trabajo ha planteado la síntesis de dos derivados de PZA que incluyen un centro metálico de cobalto (II), con la finalidad de obtener compuestos anti-tuberculosis con el potencial de actuar sobre cepas resistentes a PZA así como de reducir la generación de resistencia antibiótica. La caracterización de los complejos sintetizados es de fundamental importancia ya que no solo permiten el descubrimiento de la estructura molecular, sino también podrían favorecer el futuro descubrimiento de un mecanismo de acción probable.

Los complejos de cobalto exhiben un resultado prometedor inhibiendo el crecimiento de la bacteria en comparación con el fármaco libre (PZA) posiblemente debido a la presencia de los iones metálicos que alteran la estructura del fármaco mejorando aún más el rendimiento de su transporte, lo que es importante para aumentar la capacidad antituberculosa.

\section{PARTE EXPERIMENTAL}

\section{Síntesis del complejo [ $\left.\mathrm{Co}(\mathrm{PZA})_{2} \mathrm{Cl}_{2}\right]$}

Se disolvió 1,10 g (4,62 mmol) de cloruro de cobalto (II) hexahidratado (J.T. Baker) en 100 $\mathrm{mL}$ de metanol (Merck) en un balón de reacción. Posteriormente se agregó al mismo balón el ligando pirazinamida (Merck) 1,14 g (9,26 mmol), previamente disuelto en $100 \mathrm{~mL}$ de metanol. La síntesis se llevó a cabo en reflujo por aproximadamente dos horas en constante agitación, transcurrido el tiempo de reacción se filtró y se lavó el precipitado formado con metanol caliente.

\section{Síntesis del complejo $\left[\mathrm{Co}(\mathrm{PZA})_{2}\left(\mathrm{NO}_{3}\right)_{2}\right] \cdot \mathrm{H}_{2} \mathrm{O}$}

Se disolvió 1,51 g (5,19 mmol) de nitrato de cobalto (II) hexahidratado (Merck) en $150 \mathrm{~mL}$ de metanol (Merck) en un balón de reacción. Posteriormente, se agregó al mismo balón el ligando pirazinamida (Merck) 1,28 g (10,40 mmol), previamente disuelto en $100 \mathrm{~mL}$ de metanol. La síntesis se llevó a cabo en reflujo por aproximadamente dos horas en constante agitación, transcurrido el tiempo de reacción se filtró y se lavó el precipitado formado con metanol caliente.

\section{Técnicas de caracterización de los complejos}

\section{Análisis elemental de $\mathrm{C}, \mathrm{Hy} \mathrm{N}$}

Se determinó el porcentaje de carbono, hidrógeno y nitrógeno en cada complejo con el analizador Perkin-Elmer 2400 Series II de la Central Analítica del Instituto de Química de la Universidad de Sao Paulo.

\section{Espectroscopía de absorción atómica de cobalto}

Se determinó el contenido de cobalto en cada complejo con el espectrofotómetro de absorción atómica Shimadzu AA-7000 de la Universidad Nacional de Ingeniería, utilizando una lámpara de cátodo hueco de cobalto a $240,7 \mathrm{~nm}$. La curva de calibración se preparó en concentraciones de 0,4, 1,0 y 2,0 ppm de cobalto en ácido nítrico (Merck) 0,5 M y los complejos fueron disueltos en ácido nítrico $0,5 \mathrm{M}$ para obtener una concentración aproximada de 1 ppm de cobalto. 


\section{Determinación de cloruros en el complejo [Co $\left.(\mathrm{PZA})_{2} \mathrm{Cl}_{2}\right]$}

Se determinó el contenido de cloruros en el complejo por volumetría con el método argentométrico. Para ello, se preparó la muestra con $0,0455 \mathrm{~g}$ de complejo en un volumen final de $50 \mathrm{~mL}$ y se valoró la muestra por duplicado con nitrato de plata (Merck) 0,0481 N utilizando dicromato de potasio (Merck) como indicador.

\section{Análisis termogravimétrico}

Se realizó el análisis termogravimétrico de los complejos con el analizador TA Instruments TGA Q500 de la Central Analítica del Instituto de Química de la Universidad de Sao Paulo, la temperatura máxima de alcance fue de $950{ }^{\circ} \mathrm{C}$.

\section{Susceptibilidad magnética}

La determinación del momento magnético se realizó mediante la medición de las masas de los complejos en una balanza SHIMADZU AW120 del Laboratorio LAQISE-BIMET del Instituto de Química de la Universidad de Sao Paulo con la aplicación de un campo magnético mediante un dispositivo elaborado en el mismo laboratorio. Este análisis se realizó midiendo por cuadruplicado la masa de los complejos antes y después de la aplicación de un campo magnético. Para la verificación de las mediciones se utilizó un patrón de tetra(tiocianato) cobaltato (II) de mercurio, $\mathrm{Hg}[\mathrm{Co}(\mathrm{SCN}) 4]$ que presenta un valor de $\chi=16,44 \times 10^{-6}$ unidades CGS/Gauss a $20^{\circ} \mathrm{C}$ midiendo su masa de la misma manera que los complejos.

\section{Espectroscopía UV-Visible}

Los espectros UV-Visible de los complejos y el ligando se obtuvieron con el espectrofotómetro Shimadzu UV-1800 de la Universidad Nacional de Ingeniería, en el rango de 200 a $900 \mathrm{~nm}$. Para la medición en el rango visible se prepararon soluciones de los complejos de $0,002 \mathrm{M}$ en metanol (Merck). Mientras que, para la medición en el rango ultravioleta, las concentraciones fueron de $0,001 \mathrm{M}$, aproximadamente.

\section{Espectroscopía infrarroja FTIR}

Los espectros de infrarrojo de los complejos y el ligando fueron obtenidos por lectura directa con el espectrofotómetro Perkin-Elmer de la Universidad Nacional de Ingeniería, con reflectancia total atenuada (ATR) desde 400 a $4000 \mathrm{~cm}^{-1}$.

\section{Espectroscopía Raman}

Se obtuvieron los espectros de los complejos y el ligando con el espectrofotómetro Raman Horiba One Xplora de la Universidad Nacional de Ingeniería, utilizando un láser de $785 \mathrm{~nm}$ con una potencia de $50 \mathrm{~mW}$ desde 200 a $1800 \mathrm{~cm}-1$.

\section{Determinación de la concentración mínima inhibitoria (MIC)}

La concentración mínima inhibitoria fue estimada cultivando tres cepas de $M$. tuberculosis (H37Rv, DM97 y D49N) en caldos de cultivo 7H9 enriquecidos con OADC (albumina oleica dextrosa catalasa) a pH 6 y 7 con diferentes concentraciones de pirazinamida y complejos 6 . La cepa H37Rv es una cepa de referencia sensible a pirazinamida; las cepas DM97 y D49N son cepas aisladas de muestras de pacientes resistentes a pirazinamida. 


\section{RESULTADOS Y DISCUSIÓN}

\section{Síntesis de los complejos $\left[\mathrm{Co}(\mathrm{PZA})_{2} \mathrm{Cl}_{2}\right]$ y $\left[\mathrm{Co}(\mathrm{PZA})_{2}\left(\mathrm{NO}_{3}\right)_{2}\right] \cdot \mathrm{H}_{2} \mathrm{O}$}

De la síntesis de los complejos $\left[\mathrm{Co}(\mathrm{PZA})_{2} \mathrm{Cl}_{2}\right]$ y $\left[\mathrm{Co}(\mathrm{PZA})_{2}\left(\mathrm{NO}_{3}\right)_{2}\right] \cdot \mathrm{H}_{2} \mathrm{O}$ se obtuvieron dos sólidos no cristalinos de color lila (figura 1A) y color anaranjado (figura 1B), respectivamente. Los rendimientos de las reacciones fueron de $89 \%$ y $55 \%$, respectivamente. Además, se determinó que ambos complejos se descomponen a temperaturas mayores a 240 y 260 ${ }^{\circ} \mathrm{C}$, respectivamente. Ambos complejos son solubles en solventes polares que apolares, a excepción de la acetona, donde ambos complejos son insolubles.

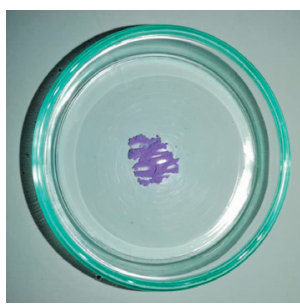

A

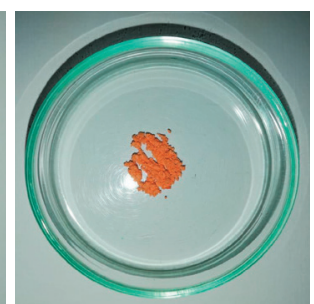

B

Figura 1. (A) Fotografía del complejo $\left[\mathrm{Co}(\mathrm{PZA})_{2} \mathrm{Cl}_{2}\right]$. (B) Fotografía del complejo $\left[\mathrm{Co}(\mathrm{PZA})_{2}\left(\mathrm{NO}_{3}\right)_{2}\right] \cdot \mathrm{H}_{2} \mathrm{O}$.

\section{Composición química elemental de los complejos}

Los resultados del análisis elemental $(\mathrm{C}, \mathrm{H}$ y N), contenido de cobalto $(\mathrm{Co})$ y cloruros se muestran a continuación en la tabla 1 , junto a los valores calculados de acuerdo con las fórmulas moleculares propuestas.

Tabla 1. Resultados de la composición elemental de los complejos.

\begin{tabular}{ccccc}
\hline \multirow{2}{*}{ Elemento } & \multicolumn{2}{c}{$\left[\mathrm{Co}(\mathrm{PZA})_{2} \mathrm{Cl}_{2}\right]$} & \multicolumn{2}{c}{$\left[\mathrm{Co}(\mathrm{PZA})_{2}\left(\mathrm{NO}_{3}\right)_{2}\right] \cdot \mathrm{H}_{2} \mathrm{O}$} \\
\cline { 2 - 5 } & \% Experimental & \% Calculado & \% Experimental & \% Calculado \\
\hline $\mathrm{C}$ & 32,39 & 31,94 & 26,01 & 26,86 \\
\hline $\mathrm{H}$ & 2,97 & 2,68 & 2,87 & 2,0 \\
\hline $\mathrm{N}$ & 22,82 & 22,35 & 24,65 & 25,06 \\
\hline $\mathrm{Co}$ & 15,62 & 15,67 & 13,13 & 13,18 \\
\hline $\mathrm{Cl}$ & 18,74 & 18,85 & No aplica & No aplica \\
\hline
\end{tabular}

\section{Análisis termogravimétrico}

El complejo $\left[\mathrm{Co}(\mathrm{PZA})_{2} \mathrm{Cl}_{2}\right]$ se descompone en tres etapas, la pérdida total de estas tres etapas fue de 79,58, dejando como residuo el 20,42 \% de la masa inicial analizada, el cual es asignado al óxido metálico formado al final del proceso ${ }^{5,7}$. De la misma manera, el complejo [Co(PZA) $\left.)_{2}\left(\mathrm{NO}_{3}\right)_{2}\right] \cdot \mathrm{H}_{2} \mathrm{O}$ se descompone en 4 etapas, formando un óxido de cobalto $^{5,7}$ al final del proceso, el cual es asignado al residuo final de 17,46 \%. En la tabla 2 se muestran los porcentajes experimental y calculado del óxido de cobalto formado ( $\mathrm{CoO})$ al final de la descomposición térmica de cada complejo. Las diferencias entre los porcentajes experimentales y calculados, puede deberse a la pureza de los complejos ${ }^{5}$. 

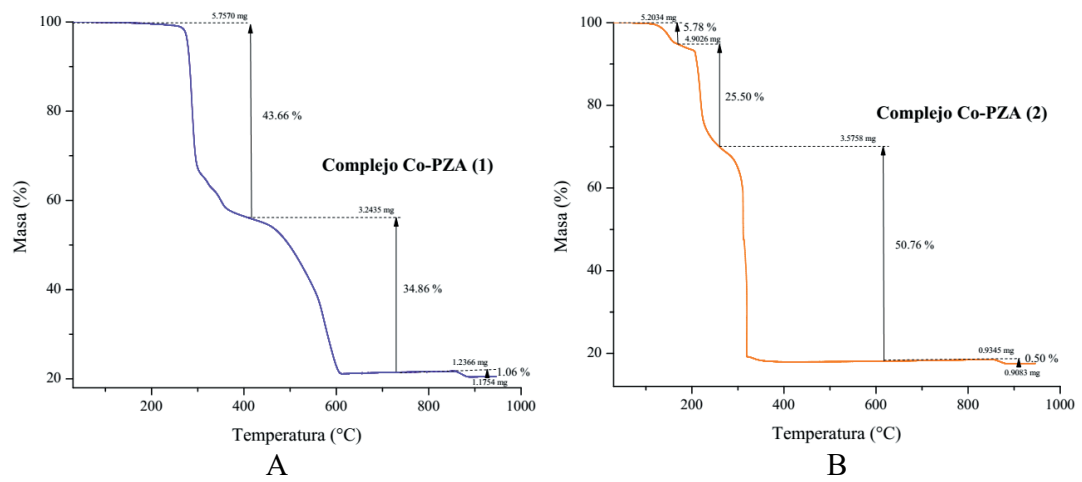

Figura 2. Curvas termogravimétricas de (A) El complejo $\left[\mathrm{Co}(\mathrm{PZA})_{2} \mathrm{Cl}_{2}\right]$

(B) El complejo $\left[\mathrm{Co}(\mathrm{PZA})_{2}\left(\mathrm{NO}_{3}\right)_{2}\right] \cdot \mathrm{H}_{2} \mathrm{O}$.

Tabla 2. Resultados del porcentaje de óxido de cobalto formado (CoO).

\begin{tabular}{ccc}
\hline Complejo & \% Experimental & \% Calculado \\
\hline$\left[\mathrm{Co}(\mathrm{PZA})_{2} \mathrm{Cl}_{2}\right]$ & 20,42 & 19,93 \\
\hline$\left[\mathrm{Co}(\mathrm{PZA})_{2}\left(\mathrm{NO}_{3}\right)_{2}\right] \cdot \mathrm{H}_{2} \mathrm{O}$ & 17,46 & 16,76 \\
\hline
\end{tabular}

\section{Susceptibilidad magnética}

Los valores del momento magnético efectivo ( $\mu$ eff) obtenido experimentalmente para los complejos $\left[\mathrm{Co}(\mathrm{PZA})_{2} \mathrm{Cl}_{2}\right]$ y $\left[\mathrm{Co}(\mathrm{PZA})_{2}\left(\mathrm{NO}_{3}\right)_{2}\right] . \mathrm{H}_{2} \mathrm{O}$ fueron de 4,668 y 4,473, respectivamente. Estos valores se obtuvieron de las medidas de susceptibilidad magnética molar corregidas con las contribuciones diamagnéticas de los iones y ligandos presentes en los complejos. Las contribuciones diamagnéticas se estimaron usando las constantes de Pascal $^{8,9}$ mostrados en la tabla 3.

Tabla 3. Resultados del porcentaje de óxido de cobalto formado (CoO).

\begin{tabular}{cc}
\hline Iones / elemento / molécula & $\begin{array}{c}\text { Contribución diamagnética } \\
\left(\times 10^{-6} \text { unidades CGS }\right)\end{array}$ \\
\hline $\mathrm{Co} 2+$ & -12 \\
\hline $\mathrm{C}$ & -6 \\
\hline $\mathrm{H}$ & -2.93 \\
\hline $\mathrm{O}$ & -1.73 \\
\hline $\mathrm{N}$ & -4.61 \\
\hline $\mathrm{Cl}^{-}$ & -23 \\
\hline $\mathrm{NO}_{3} \mathrm{H}_{2} \mathrm{O}$ & -19 \\
\hline
\end{tabular}


Con base en los valores del momento magnético efectivo obtenidos de los complejos y de acuerdo con la tabla 4, se comprueba que ambos valores corresponden al ion metálico de $\mathrm{Co}^{2+}$ con tres electrones desapareados en un entorno octaédrico ${ }^{8,10}$, es decir, un campo octaédrico débil o de espín alto con configuración $t_{2 g}^{5} e_{g}^{2}$ y EECC (energía de estabilización de campo cristalino) igual a $0.8 \Delta \mathrm{o}^{8}$.

Tabla 4. Momento magnético para algunos iones de metales de transición ${ }^{17}$.

\begin{tabular}{ccc}
\hline Iones & $\begin{array}{c}\mu_{\text {eff }} \\
\text { teórico o calculado }\end{array}$ & $\begin{array}{c}\mu_{\text {eff }} \\
\text { experimental }\end{array}$ \\
\hline $\mathrm{V}^{4+}$ & 1,73 & $1,7-1,8$ \\
\hline $\mathrm{Cu}^{2+}$ & 1,73 & $1,7-2,2$ \\
\hline $\mathrm{V}^{3+}$ & 2,83 & $2,6-2,8$ \\
\hline $\mathrm{Ni}^{2+}$ & 2,83 & $2,8-4,0$ \\
\hline $\mathrm{Cr}^{3+}$ & 3,87 & $\sim 3,8$ \\
\hline $\mathrm{Co}^{2+}$ & 3,87 & $4,1-5,2$ \\
\hline $\mathrm{Fe}^{2+}$ & 4,90 & $5,1-5,5$ \\
\hline $\mathrm{Co}^{3+}$ & 4,90 & $\sim 5,4$ \\
\hline $\mathrm{Mn}^{2+}$ & 5,92 & $\sim 5,9$ \\
\hline $\mathrm{Fe}^{3+}$ & 5,92 & $\sim 5,9$ \\
\hline
\end{tabular}

\section{Espectroscopía UV- Visible}

El diagrama de Orgel para el ion $\mathrm{Co}^{2+}$ en campos octaédricos débiles revela que se debe observar un espectro con tres absorciones ${ }^{8}$. Transiciones desde el estado basal: ${ }^{4} \mathrm{~T}_{1 \mathrm{~g}}(\mathrm{~F})$, a ${ }^{4} \mathrm{~T}_{2 \mathrm{~g}}(\mathrm{~F}),{ }^{4} \mathrm{~A}_{2 \mathrm{~g}}(\mathrm{~F}) \mathrm{y}{ }^{4} \mathrm{~T}_{1 \mathrm{~g}}(\mathrm{P})$ provocan absorciones de banda ancha en la región del visible $y$ el infrarrojo cercano. La primera banda, asignada a la transición ${ }^{4} \mathrm{~T}_{1 \mathrm{~g}}(\mathrm{~F}) \leftrightarrow{ }^{4} \mathrm{~T}_{2 \mathrm{~g}}(\mathrm{~F})$, es usualmente encontrada en la región de $5500-11000 \mathrm{~cm}^{-1}$. La segunda banda es débil (pero permitida por el spin), asignada a la transición ${ }^{4} \mathrm{~T}_{1 \mathrm{~g}}(\mathrm{~F}) \leftrightarrow{ }^{4} \mathrm{~A}_{2 \mathrm{~g}}(\mathrm{~F})$ es encontrada en la región de $12000-17000 \mathrm{~cm}^{-1}$; la tercera banda es asignada a la transición ${ }^{4} \mathrm{~T}_{1 \mathrm{~g}}(\mathrm{~F}) \leftrightarrow{ }^{4} \mathrm{~T} 1 \mathrm{~g}(\mathrm{P}),{ }^{2} \mathrm{P},{ }^{2} \mathrm{G}$ y usualmente es encontrada en la región de $16000-22000 \mathrm{~cm}^{-1}{ }^{11}$. Los espectros UV-Visible de los complejos muestran dos bandas para cada complejo, a $320 \mathrm{~nm}\left(31250 \mathrm{~cm}^{-1}\right)$ y $522 \mathrm{~nm}$ $\left(19157 \mathrm{~cm}^{-1}\right)$ para el complejo $\left[\mathrm{Co}(\mathrm{PZA})_{2} \mathrm{Cl}_{2}\right.$; ; y a $319 \mathrm{~nm}\left(31347 \mathrm{~cm}^{-1}\right)$ y $513 \mathrm{~nm}(19493$ $\mathrm{cm}^{-1}$ ) para el complejo $\left[\mathrm{Co}(\mathrm{PZA})_{2}\left(\mathrm{NO}_{3}\right)_{2}\right] \cdot \mathrm{H}_{2} \mathrm{O}$. Las bandas a 320 y $319 \mathrm{~nm}$ (ultravioleta) de los complejos se atribuyen a la presencia del ligando debido a las transiciones electrónicas de tipo $\pi \rightarrow \pi^{* 12}$ y las bandas a 522 y $513 \mathrm{~nm}$ (visible) a la presencia del metal de transición. Estas dos últimas, son bandas de baja absorbancia opacadas por la intensidad de las bandas en la región del ultravioleta, y son asignadas a la transición 4T1g $(\mathrm{F}) \leftrightarrow 4 \mathrm{~T} 1 \mathrm{~g}(\mathrm{P})$; debido a que las transiciones ${ }^{4} \mathrm{~T}_{1 \mathrm{~g}}(\mathrm{~F}) \leftrightarrow{ }^{4} \mathrm{~T}_{2 \mathrm{~g}}(\mathrm{~F}) \mathrm{y}{ }^{4} \mathrm{~T}_{1 \mathrm{~g}}(\mathrm{~F}) \leftrightarrow{ }^{4} \mathrm{~A}_{2 \mathrm{~g}}(\mathrm{~F})$ ocurren a menor número de onda y no se observan en los espectros.

\section{Espectroscopía infrarroja FTIR}

Pirazinamida tiene cuatro átomos donadores: los nitrógenos del anillo pirazina, el oxígeno del grupo carbonilo y el nitrógeno del grupo amino, por lo tanto, tiene diferentes modos de enlace al ion metálico ${ }^{13}$, es decir diferentes sitios de coordinación (figura 3). En algunos complejos, la coordinación de la pirazinamida al metal ocurre a través de los nitrógenos del anillo $^{12}$ y otras veces a través del oxígeno de grupo carbonilo ${ }^{14}$. 


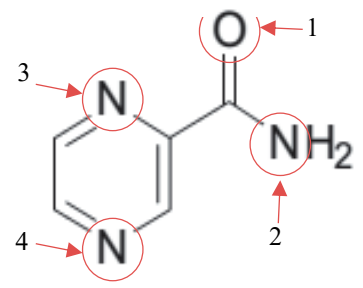

Figura 3. Molécula de pirazinamida y sus posibles modos de coordinación.

$\mathrm{Al}$ comparar los espectros de pirazinamida (figura 4) y el complejo $\left[\mathrm{Co}(\mathrm{PZA})_{2} \mathrm{Cl}_{2}\right]$ (figura 5), se observa que hay cambios en los números de onda de los picos relacionados con el grupo funcional amida $\mathrm{NH}_{2}$ y el anillo aromático. Los cambios más resaltantes se observan en los dos primeros picos de pirazinamida $\left(3408\right.$ y $3148 \mathrm{~cm}^{-1}$ ) que corresponden al $\mathrm{NH}_{2}$ debido a la tensión asimétrica y simétrica de los enlaces $\mathrm{N}-\mathrm{H}^{15}$, además también se observa que el pico de $1608 \mathrm{~cm}^{-1}$ que corresponde a la flexión de los enlaces N-H sufre un desplazamiento a 1591 $\mathrm{cm}^{-1}$ en el complejo. Así también, se observan desplazamientos y cambios morfológicos en los picos a 618, 511 y $428 \mathrm{~cm}^{-1}$ de pirazinamida hacia 639,531 y $451 \mathrm{~cm}^{-1}$ en el complejo $\left[\mathrm{Co}(\mathrm{PZA})_{2} \mathrm{Cl}_{2}\right]$ que corresponden a vibración de torsión, balanceo y flexión fuera del plano del grupo amida ${ }^{16}$. Todos estos cambios observados en el rango de 3500-3100, 1600 y de 600-400 $\mathrm{cm}^{-1}$, aproximadamente evidencian la formación de un enlace a través del nitrógeno del grupo amida con el centro metálico de cobalto. Por otro lado, también se observan desplazamientos en los picos que han sido asignados al anillo aromático ${ }^{16}$ y se observa además la aparición o intensificación en el espectro del complejo $\left[\mathrm{Co}(\mathrm{PZA})_{2} \mathrm{Cl}_{2}\right]$ de una vibración a $949 \mathrm{~cm}^{-1}$ que no está activa en el espectro de pirazinamida. De acuerdo con la bibliografía revisada este pico puede corresponder a una vibración del anillo aromático ${ }^{17}$ que tiene sustituciones en las posiciones 1,2 y 4 (del anillo), es decir, un anillo bencénico 1,2 y 4 - trisustituido $^{15}$ que además tiene otra vibración a $780 \mathrm{~cm}^{-1}$ y que se expresa a 785 y $793 \mathrm{~cm}^{-1}$ en pirazinamida y el complejo $\left[\mathrm{Co}(\mathrm{PZA})_{2} \mathrm{Cl}_{2}\right]$, respectivamente. Por lo tanto, un enlace a través del nitrógeno en la posición 4 del anillo (tomando como posición 2 la sustitución debido al grupo amida) y el centro metálico de cobalto podría tomar lugar.
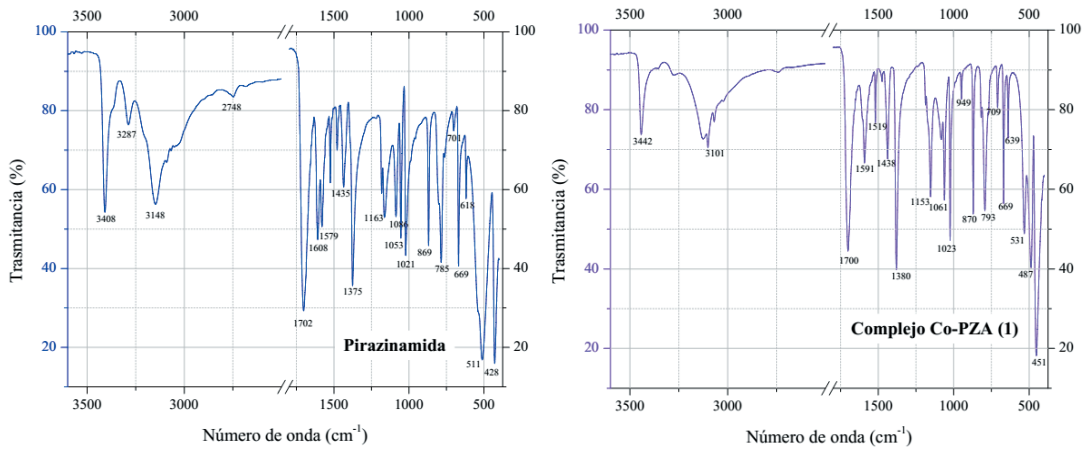

Figura 3. Espectro IR de pirazinamida. Figura 5. Espectro IR de $\left[\mathrm{Co}(\mathrm{PZA})_{2} \mathrm{Cl}_{2}\right]$. 
Para el complejo $\left[\mathrm{Co}(\mathrm{PZA})_{2}\left(\mathrm{NO}_{3}\right)_{2}\right] \cdot \mathrm{H}_{2} \mathrm{O}$ (figura 6$)$, se observa que hay cambios en los números de onda y morfología de los picos relacionados con el grupo funcional amida $\mathrm{NH}_{2}$ y carbonilo $\mathrm{C}=\mathrm{O}$. Si empezamos el análisis por las bandas a mayor número de onda encontramos que los picos a 3369 y $3086 \mathrm{~cm}^{-1}$ del complejo, corresponden a los desplazamientos de los picos a 3408 y $3148 \mathrm{~cm}^{-1}$ de pirazinamida, vibraciones que se deben a la tensión simétrica y asimétrica del enlace $\mathrm{N}-\mathrm{H}^{15}$. También se observa que el pico a $3287 \mathrm{~cm}^{-1}$ de pirazinamida debido a la tensión simétrica del enlace $\mathrm{N}-\mathrm{H}^{16}$, no aparece en el espectro del complejo $\left[\mathrm{Co}(\mathrm{PZA})_{2}\left(\mathrm{NO}_{3}\right)_{2}\right]$. $\mathrm{H}_{2} \mathrm{O}$. Además, el pico a $1608 \mathrm{~cm}^{-1}$ que corresponde a la flexión de los enlaces $\mathrm{N}-\mathrm{H}^{16}$ sufre un desplazamiento a $1624 \mathrm{~cm}^{-1}$, así como la vibración a $1086 \mathrm{~cm}^{-1}$ por balanceo del grupo amida $\mathrm{NH}_{2}$ se desplaza a $1098 \mathrm{~cm}^{-1}$. Todos estos cambios mencionados anteriormente reflejan la formación de un enlace a través del nitrógeno del grupo amida y el centro metálico de cobalto del complejo $\left[\mathrm{Co}(\mathrm{PZA})_{2}\left(\mathrm{NO}_{3}\right)_{2}\right] \cdot \mathrm{H}_{2} \mathrm{O}$. Otra variación importante entre los espectros es la banda a $1683 \mathrm{~cm}^{-1}$ del complejo Co-PZA (2) proveniente del desplazamiento del pico a $1702 \mathrm{~cm}^{-1}$ de pirazinamida. El rango general para verificar la presencia de vibraciones debido al grupo funcional $\mathrm{C}=\mathrm{O}$ es de $1850-1550 \mathrm{~cm}^{-1}$ debido a la tensión entre el carbono y el oxígeno de la molécula ${ }^{15}$, siendo esta su banda característica. Por lo tanto, se asigna al pico de $1702 \mathrm{~cm}^{-1}$ la vibración del enlace $\mathrm{C}=\mathrm{O}^{16}$ en pirazinamida y al pico de $1683 \mathrm{~cm}^{-1}$ del complejo. Al verificar un desplazamiento en el espectro del complejo para la vibración más representativa de este grupo, se puede afirmar que a través del oxígeno se estaría dando lugar a un enlace con el centro metálico del complejo. Otras zonas importantes en el espectro del complejo $\left[\mathrm{Co}(\mathrm{PZA})_{2}\left(\mathrm{NO}_{3}\right)_{2}\right] \cdot \mathrm{H}_{2} \mathrm{O}$ que no provienen de las vibraciones causadas por los grupos funcionales del ligando pirazinamida evidencian variaciones. La primera de ellas se da en la región de 1500-1250 $\mathrm{cm}^{-1}$ donde se observan bandas a 1406, 1386, 1309 y 1296 $\mathrm{cm}^{-1}$. En esta zona ya existen vibraciones provenientes de pirazinamida, sin embargo, de acuerdo con la literatura, también en esta zona se expresan vibraciones debido al enlace

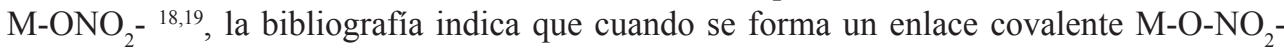
se origina un desdoblamiento de dos bandas presentes en el espectro del ion nitrato, el primer desdoblamiento ocurre con una banda aproximadamente a $1390 \mathrm{~cm}-1$ la cual genera bandas a 1300 y $1500 \mathrm{~cm}^{-1}$ aproximadamente ${ }^{18,19}$. El desdoblamiento de la banda del ion nitrato al formarse el enlace $\mathrm{M}-\mathrm{O}-\mathrm{NO}_{2}$ - ocurre a 1406 y $1309-1296 \mathrm{~cm}^{-1}$ en el espectro del complejo $\left[\mathrm{Co}(\mathrm{PZA})_{2}\left(\mathrm{NO}_{3}\right)_{2}\right] \cdot \mathrm{H}_{2} \mathrm{O}$. El segundo desdoblamiento ocurre con una banda a 720 $\mathrm{cm}^{-1}$ (perteneciente al ion nitrato) la cual genera bandas débiles que muchas veces pasan desapercibidas en el espectro infrarrojo ${ }^{18}$. En el espectro del complejo $\left[\mathrm{Co}(\mathrm{PZA})_{2}\left(\mathrm{NO}_{3}\right)_{2}\right]$. $\mathrm{H}_{2} \mathrm{O}$ existen bandas a 714 y $702 \mathrm{~cm}^{-1}$ las cuales son consideradas señales producto del desdoblamiento de la banda $720 \mathrm{~cm}^{-1} 18,19$ del ion nitrato. Por lo tanto, de acuerdo con las variaciones observadas, se deduce que la coordinación del ligando pirazinamida hacia el átomo metálico de cobalto sucede a través del nitrógeno y oxígeno del grupo amida, ocupando cuatro sitios de coordinación en total por las dos moléculas de pirazinamida presentes en el complejo. Además, también se evidencia la formación de un enlace covalente a través del oxígeno del grupo nitrato de forma monodentada. 


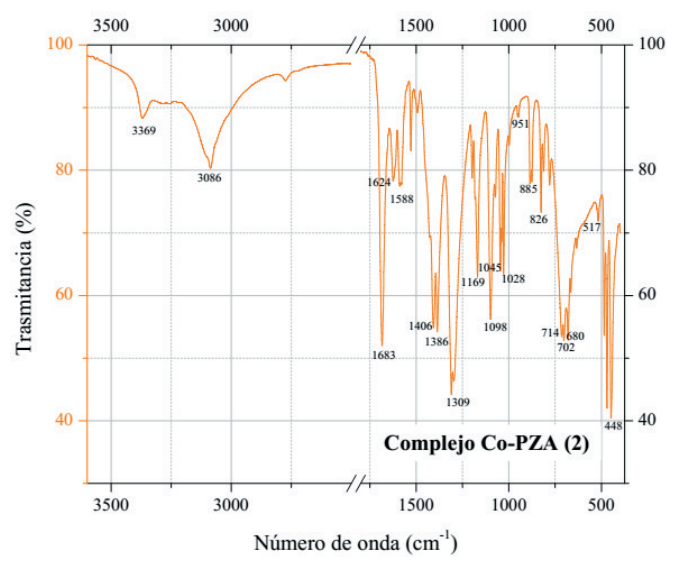

Figura 6. Espectro IR de $\left[\mathrm{Co}(\mathrm{PZA})_{2}\left(\mathrm{NO}_{3}\right)_{2}\right] \cdot \mathrm{H}_{2} \mathrm{O}$.

\section{Espectroscopía Raman}

Se analizaron las variaciones en los números de onda de los picos más importantes del espectro de pirazinamida (figura 7) y de los complejos. Se observa dos picos en el espectro del complejo [Co(PZA) $\mathrm{Cl}_{2}$ ] (figura 8) que no aparecen en el espectro de pirazinamida, el primero a $225 \mathrm{~cm}^{-1}$ y el segundo a $454 \mathrm{~cm}^{-1}$, de acuerdo con la bibliografía revisada para

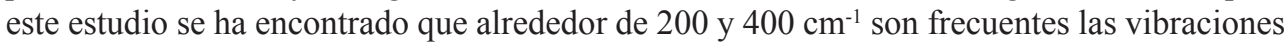
del enlace $\mathrm{M}-\mathrm{Cl}^{17}$. Por ejemplo, a $430 \mathrm{~cm}^{-1}$ se observa una banda para el compuesto de $\mathrm{CoCl}_{2}$, además a 320 y $230 \mathrm{~cm}^{-1}$ se observan bandas para los compuestos de $\mathrm{CoCl}_{2} .2$ py y $\mathrm{CoCl}_{2} .4$ py, respectivamente (donde py representa a piridina) ${ }^{15}$. Por otro lado, J. E. Rüede y D. A. Thornton ${ }^{20}$ afirman que para un compuesto octaédrico de cobalto del tipo $\mathrm{ML}_{2} \mathrm{X}_{2}$ donde L es piridina y $\mathrm{X}$ es cloro, existen dos vibraciones en el infrarrojo correspondientes al enlace $\mathrm{Co}-\mathrm{Cl}$ los cuales aparecen a 222 y $230 \mathrm{~cm}^{-1}$. Mientras que para un compuesto de tipo tetraédrico las vibraciones para el mismo enlace aparecen a 307 y $346 \mathrm{~cm}^{-1} 20$. Por lo tanto, con la evidencia de los rangos a los que aparecen las vibraciones de un enlace tipo $\mathrm{M}-\mathrm{X}$ (donde $\mathrm{M}$ es el metal y X es el halógeno) tanto en el espectro infrarrojo como en el Raman, en este estudio se ha asignado al enlace $\mathrm{Co}-\mathrm{Cl}$ los picos de 225 y $454 \mathrm{~cm}^{-1}$ del complejo. También se observan diferencias importantes en los números de onda que corresponden a los enlaces C-C, C-H, C-N del anillo aromático. Por ejemplo, el pico a $779 \mathrm{~cm}^{-1}$ en pirazinamida se presenta como una señal de baja intensidad a $765 \mathrm{~cm}^{-1}$ en el complejo, esta vibración es asignada al enlace $\mathrm{C}-\mathrm{N}$ del anillo ${ }^{16}$. Otro cambio que se observa en cuanto a las vibraciones generadas por el anillo aromático es el alejamiento entre los picos a 1168 y $1182 \mathrm{~cm}^{-1} \mathrm{de}$ pirazinamida que en el complejo aparecen a 1160 y $1188 \mathrm{~cm}^{-1}$ duplicando su separación. Por las diferencias observadas entre los espectros que corresponden a pirazinamida y el complejo $\left[\mathrm{Co}(\mathrm{PZA})_{2} \mathrm{Cl}_{2}\right]$, en este estudio se concluye que existen enlaces entre el centro metálico de cobalto y el cloro $(\mathrm{Co}-\mathrm{Cl})$ y además con un nitrógeno del grupo amida y del anillo aromático (Co-N). 


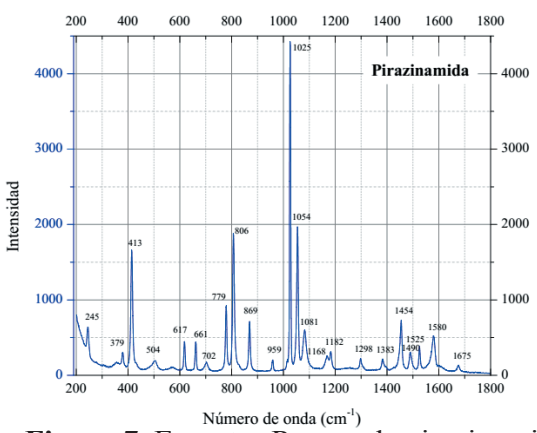

Figura 7. Espectro Raman de pirazinamida.

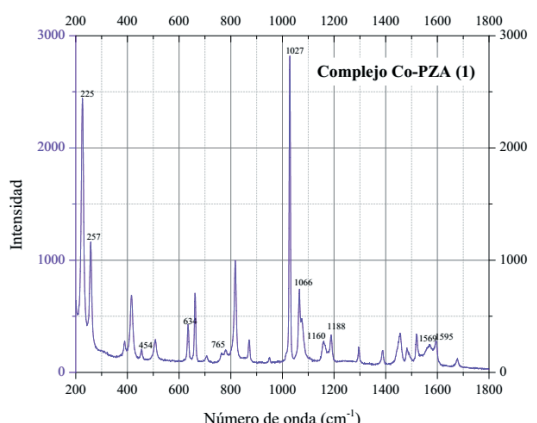

Figura 8. Espectro Raman de $\left[\mathrm{Co}(\mathrm{PZA})_{2} \mathrm{Cl}_{2}\right]$.

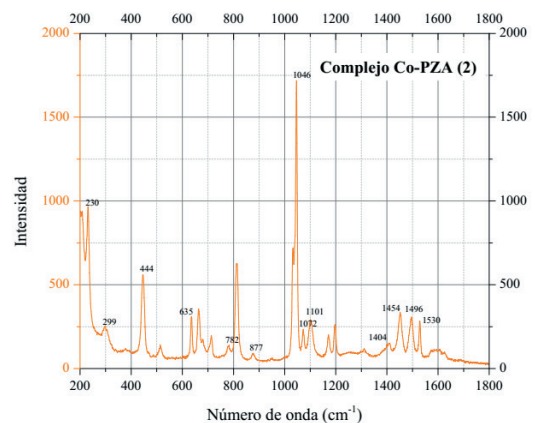

Figura 9. Espectro Raman del complejo $\left[\mathrm{Co}(\mathrm{PZA})_{2}\left(\mathrm{NO}_{3}\right)_{2}\right] \cdot \mathrm{H}_{2} \mathrm{O}$.

Al comparar los espectros de pirazinamida y el complejo $\left[\mathrm{Co}(\mathrm{PZA})_{2}\left(\mathrm{NO}_{3}\right)_{2}\right] \cdot \mathrm{H}_{2} \mathrm{O}$ (figura 9), se observa que los cambios más significativos ocurren con las absorciones relacionadas al grupo amida como los desplazamientos de las señales de pirazinamida a 413, 617 y $1081 \mathrm{~cm}^{-1}{ }^{18}$ hacia 444, 635 y $1101 \mathrm{~cm}^{-1}$ en el complejo. Otro cambio importante se observa en el pico a 1675 
$\mathrm{cm}^{-1}$ de pirazinamida, este pico es característico del grupo $\mathrm{C}=\mathrm{O}^{16}$ y se observa el cambio de su señal en el espectro del complejo $\left[\mathrm{Co}(\mathrm{PZA})_{2}\left(\mathrm{NO}_{3}\right)_{2}\right] \cdot \mathrm{H}_{2} \mathrm{O}$. De esta manera, se evidencia la formación de un enlace entre el cobalto y el oxígeno del grupo $\mathrm{C}=\mathrm{O}$. Por lo tanto, luego de analizar los cambios importantes entre los espectros de pirazinamida y el complejo, se concluye que los enlaces entre el centro metálico de cobalto y la molécula de pirazinamida suceden a través del nitrógeno del grupo amida y del oxígeno del mismo grupo.

Finalmente, en las figuras 10 (A y B) se muestran las estructuras propuestas basadas en la caracterización realizada.<smiles></smiles>

A

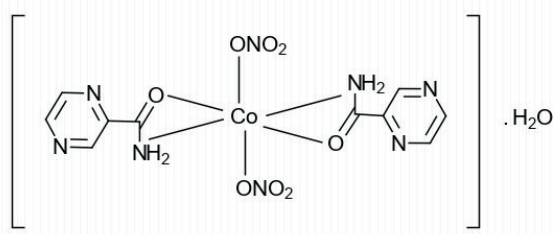

B

\section{Determinación de la concentración mínima inhibitoria (MIC)}

Los resultados de la determinación de la MIC de pirazinamida y los complejos se muestran en la tabla 5 .

Tabla 5. Resultados de la concentración mínima inhibitoria de pirazinamida y los complejos.

\begin{tabular}{ccccccc}
\hline \multirow{2}{*}{ Droga } & \multicolumn{3}{c}{ pH 6 } & \multicolumn{3}{c}{ pH 7 } \\
\cline { 2 - 7 } & H37Rv & DM97 & D49N & H37Rv & DM97 & D49N \\
\hline Pirazinamida & $0,81 \mathrm{mM}$ & $>6,5 \mathrm{mM}$ & $>6,5 \mathrm{mM}$ & $>6,5 \mathrm{mM}$ & $>6,5 \mathrm{mM}$ & $>6,5 \mathrm{mM}$ \\
\hline$\left[\mathrm{Co}(\mathrm{PZA})_{2} \mathrm{Cl}_{2}\right]$ & $0,04 \mathrm{mM}$ & $0,33 \mathrm{mM}$ & $0,02 \mathrm{mM}$ & $0,33 \mathrm{mM}$ & $0,33 \mathrm{mM}$ & $0,02 \mathrm{mM}$ \\
\hline$\left[\mathrm{Co}(\mathrm{PZA})_{2}\left(\mathrm{NO}_{3}\right)_{2}\right]$ & $0,03 \mathrm{mM}$ & $0,28 \mathrm{mM}$ & $0,02 \mathrm{mM}$ & $0,28 \mathrm{mM}$ & $0,28 \mathrm{mM}$ & $0,02 \mathrm{mM}$ \\
\hline
\end{tabular}

La estimación cuantitativa de la MIC fue determinada usando el método de ensayo en microplaca con tetrazolium (TEMA), donde la capacidad de oxidación-reducción del colorante tetrazolium permitió los cambios colorimétricos con los que fue posible realizar la cuantificación de la $\mathrm{MIC}^{6}$. La tabla 5 muestra que los complejos inhiben el crecimiento de las cepas de $M$. tuberculosis sensible (H37Rv) y resistentes a pirazinamida (DM97 y D49N) tanto a pH 6 como a pH 7 en menor concentración que el fármaco libre pirazinamida, es decir, que en el ensayo en microplaca los complejos demostraron poseer mayor capacidad antimicrobiana que el fármaco libre pirazinamida, siendo el complejo $\left[\mathrm{Co}(\mathrm{PZA})_{2} \mathrm{Cl}_{2}\right]$ el de mayor actividad contra $M$. tuberculosis in vitro. 


\section{CONCLUSIONES}

Las fórmulas moleculares propuestas con base en los resultados de la caracterización para los complejos fueron $\left[\mathrm{Co}(\mathrm{PZA})_{2} \mathrm{Cl}_{2}\right]$ para el complejo Co-PZA (1) y $\left[\mathrm{Co}(\mathrm{PZA})_{2}\left(\mathrm{NO}_{3}\right)_{2}\right] \cdot \mathrm{H}_{2} \mathrm{O}$ para el complejo Co-PZA (2), en ambos complejos el centro metálico de cobalto se encuentra en un entorno octaédrico donde las moléculas de pirazinamida se ubican en el plano horizontal y los ligandos cloro y nitrato en el plano axial de la molécula. La determinación de la concentración mínima inhibitoria analizada para los complejos y el fármaco libre pirazinamida en cultivos de Mycobacterium tuberculosis en las cepas sensible (H37Rv) y resistentes a pirazinamida (DM97 y D49N) mostró que los complejos tienen mayor capacidad antimicrobiana que el fármaco libre (pirazinamida), siendo el complejo $\left[\mathrm{Co}(\mathrm{PZA})_{2} \mathrm{Cl}_{2}\right]$ el de mayor actividad contra M. tuberculosis.

\section{AGRADECIMIENTO}

Se hace un agradecimiento especial al Laboratorio de LAQISE-BIMET del Instituto de Química de la Universidad de Sao Paulo, a la Central Analítica del Instituto de Química de la Universidad de Sao Paulo y al Laboratorio de Electroquímica Aplicada (GIEA) de la Universidad Nacional de Ingeniería, por contribuir con la realización de esta investigación.

\section{REFERENCIAS BIBLIOGRÁFICAS}

1. Bouz G, Semelková L, Jand'ourek O, Konečná K, Paterová P, Navrátilová L, et al. Derivatives of 3-Aminopyrazine-2-carboxamides: Synthesis, antimicrobial evaluation, and in vitro cytotoxicity. Molecules. 2019;24(7):1212. doi: 10.3390/molecules24071212.

2. OMS. Informe Mundial sobre la Tuberculosis. [Internet] 2019. [Citado 12 mayo 2020]. Disponible en: https:/www.who.int/tb/publications/global_report/es/

3. Reddyrajula R, Dalimba U. The bioisosteric modification of pyrazinamide derivatives led to potent antitubercular agents: Synthesis via click approach and molecular docking of pyrazine-1,2,3-triazoles. Bioorganic Med Chem Lett. 2020; 30(2):126-146.

4. Ali M, Ul Qader SA, Shahid F, Arayne MS, Mumtaz M. Synthesis, characterization and enzyme inhibitory activity of new pyrazinamide iron complexes. Pak J Pharm Sci. 2017; 30(3): $825-31$.

5. Ali AE, Elasala GS, Mohamed EA, Kolkaila SA. Spectral, thermal studies and biological activity of pyrazinamide complexes. Heliyon. 2019; 5(11):e02912. doi: 10.1016/j. heliyon.2019.e02912

6. Caviedes L, Delgado J, Gilman H. Tetrazolium Microplate Assay as a Rapid and Inexpensive Colorimetric Method for Determination of Antibiotic Susceptibility of Mycobacterium tuberculosis. J Clin Microbiol. 2002 May;40(5): 1873-1874.

7. Gabbott P. Principles and Applications of Thermal Analysis. First edition. Oxford, United Kingdom: Blackwell Publishing; 2007.

8. Huheey JE, Keiter EA, Keiter RL. Inorganic Chemistry: Principles of Structure and Reactivity. 4th Edition. New York: Harper Collins; 1993. 
9. Bain GA, Berry JF. Diamagnetic Corrections and Pascal's Constants. J Chem Educ. 2008; 85(4):532-536.

10. Netalkar PP, Netalkar SP, Budagumpi S, Revankar VK. Synthesis, crystal structures and characterization of late first row transition metal complexes derived from benzothiazole core: anti-tuberculosis activity and special emphasis on DNA binding and cleavage property. Eur J Med Chem. 2014;79: 47-56.

11. Reedijk J, Driessen WL, Groeneveld WL. A semi-empirical energy-level diagram for octahedral cobalt(II) complexes. Recl Trav Chim Pays-Bas. 2010; 88(9): 1095-1109.

12. Chiniforoshan H, Radani ZS, Tabrizi L, Tavakol H, Sabzalian MR, Mohammadnezhad $\mathrm{G}$, et al. Pyrazinamide drug interacting with $\mathrm{Co}(\mathrm{III})$ and $\mathrm{Zn}(\mathrm{II})$ metal ions based on 2,2'-bipyridine and 1,10-phenanthroline ligands: Synthesis, studies and crystal structure, DFT calculations and antibacterial assays. J Mol Struct. 2015; 1081: 237-243.

13. Akyuz S, Andreeva L, Minceva-Sukarova B, Basar G. Vibrational spectroscopic study of two dimensional polymer compounds of pyrazinamide. J Mol Struct. 2007; 834-836: 399-402.

14. Shirvan SA, Haydari Dezfuli S. Catena-Poly[[[aqua(pyrazine-2-carboxamide- $\kappa 2 \mathrm{~N} 1, \mathrm{O}$ )

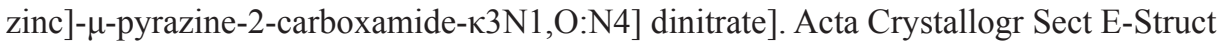
Rep Online. 2012; 68(5): 627-628.

15. Socrates G. Infrared and Raman Characteristic Group Frequencies. Hoboken, New Jersey: Wiley; 2004.

16. Chiş V, Pîrna u A, Jurca T, Vasilescu M, Simon S, Cozar O, et al. Experimental and DFT study of pyrazinamide. Chem Phys. 2005; 316(1-3): 153-163.

17. Alves OL, Hase Y. The Infrared Spectra of Cobalt(II) Halide Complexes with Trimethylphosphine Oxide (TMPO). Spectrosc Lett. 1982; 15(6): 423-433.

18. Moeller T. Inorganic Syntheses. Volume V. First edition. New York: Mcgraw-Hill Inc; 1957.

19. Curtis NF, Curtis YM. Some Nitrato-Amine Nickel(II) Compounds with Monodentate and Bidentate Nitrate Ions. Inorg Chem. 1965; 4(6): 804-809.

20. Rüede JE, Thorton DA. The far infrared spectra of metal chloride complexes of pyridine in relation to their structures. J Mol Struct. 1976; 34(1): 75-81. 